\title{
An Image Compression Using Multilayer Wavelet Transform with 2DTCWT: A Review
}

\author{
Priya Pareek \\ Department of Information \& Technology \\ Laxmi Narayan College of Technology, \\ Bhopal
}

\author{
Manish Shrivastava, Ph.D \\ Department of Information \& Technology \\ Laxmi Narayan College of Technology, \\ Bhopal
}

\begin{abstract}
Image compression is reducingsize of images in bytes of an imagewithout degrading the quality of pixelspresent in images to an unacceptable level. Whenever images are resized then it provides storage space to the other files. In this paper we have discussed a new approach for image compression, where multilayer wavelet is to be used, by using dual tree complex wavelet transform with multilayer that preserve the dominant brightness level and intensity of the targeted image in layers, which results in layered wavelet coefficients close to zero. The Thresholding also can modify the coefficients to produce more zeros which allow a higher compression ratio. The wavelet analysis needs addition support for compressing the data so Huffman coding is used along with wavelet analysis of an image in order to compress the data. Finally get the best result with higher psnr and compression ratio and minimum mse in the compared proposed.
\end{abstract}

\section{Keywords}

Image compression, JPEG2000, Wavelet Transform, DTCWT,multimedia.

\section{INTRODUCTION}

Image compression is an application of data compression that transforms the original image with minute bits. The aim of image compression is to lessen the redundancy of the image and to store or broadcast data in an efficient form. The Figure 1shows the block diagram of the general image compression system. The centralgoal of such system is to lessen the storage quantity as much as achievable and the decoded image displayed in the monitor can be analogous to the original image as much as can be. Due to demand in bandwidth and to achieve less storage capacity compression techniques like jpeg, jpeg2000 had been implemented.Wavelet Transform [1] has received more significant concentration in signal compression. Conversely, many differences lie down in the performance of different wavelets. There is necessitatefor selecting the optimal matched wavelet bases to examine the signal and the signal needs to be expressed with the fewest coefficients, i.e. sparse coefficients. The signal compression with wavelet is a procedure in which the input signal is expressed with a sum of a few of power terms for wavelet function. The more similar the bases function is to input signal, the higher the compression ratio is. But, at higher compression ratios we may experience more errors, i.e. mean square error will be high at the receiving end and hence PSNR will be very low. The discrete cosine transforms (DCT) is atechnique for converting a signal into elementary frequency components. It is widely used in image compression. There has been developed some simple functions to work out the DCT and to compress images [2]. These functions demonstrate the power of Mathematical in the prototyping of image processing algorithm.
Figure 1 shows the basic working of JPEG image compression, where each block contains useful information and encoding/decoding blocks play an important role for the compression and its reverse process.

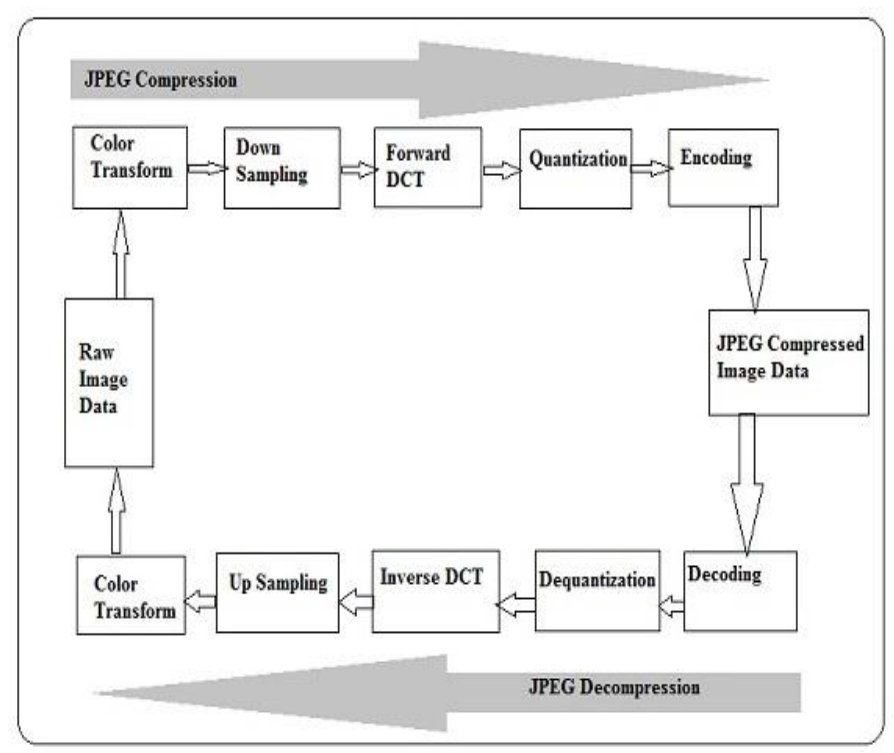

Fig. 1 General block diagram of JPEG compression

The organization of the paper is as follows: next section contains literature of the previous works done after that various image compression techniques are discussed with their advantages and demerits and finally conclusion of the presented paper.

\section{RELATED WORK}

For the image compression various author/ researcher has developed and proposed different techniques in which the review of some them are described below:

HuanjingYue et al has proposed a novel image compression schemebased on the local feature descriptor - Scale Invariant Feature Transform (SIFT). The SIFT descriptor characterizes an image region invariantly to extent and rotation. It is used extensively in image retrieval. By using SIFT descriptors; our compression scheme is able to make use of external image contents to lessen visual redundancy amongst images. The proposed encoder compresses an input image by SIFT descriptors rather than pixel values. It splits the SIFT descriptors of the image into two group; a visual description which is a considerably sub-sampled image with key SIFT descriptors embedded and a group of differential SIFT descriptors, to lessen the coding bits. Thesubsequent decoder generates the SIFT descriptors from the visual explanation and 
the differential set. The SIFT descriptors are used in our SIFTbased matching to reclaim the candidate predictive patches from a large image dataset. These contender patches are then integrated into the visual depiction presenting the final reconstructed images. Our precluding but promising results reveal the effectiveness of our proposed image coding method towards perceptual quality. This proposed image compression scheme provides a feasible approach to make use of the visual correlation among images[3].Merav Huber-Lerner et al. proposed the PCA-DCT (principle component analysis followed by discrete cosine transform) compression method. It merges the PCA capability to take out the background from a minute number of components with the individual spectral compression of each pixel of the enduring image using quantized DCT coefficients. The compression method is reserveduncomplicated for fast processing and accomplishment which considers lossy compression only on the spectral axis. It attains compression ratio of over 20 while using only spectral compression (before applying spatial compression and bit-stream-encoding). The popular RX (Reed Xiaoli) algorithm and the improved quazilocal RX (RXQLC) are used as object detection methods. The revealing performance is evaluated using ROC (receiver operating characteristics) curve generation. The proposed compression scheme shows improved detection performance compared to the detection performance of the novel image, and two other compression methods: PCA-ICA and band decimation [4]. G Boopathiet al.Proposed approach is a popular neural network method called Radial Basis Function (RBF) approach is used to produce the code book. A shared approach of image compression based on vector quantization and wavelet transform is proposed using RBF neural network. This method will be very helpful for medical imaging, criminal inquiry where high exactituderefurbish image is required. An experimental result shows that the proposed technique provides better PSNR value and also reduces the Mean Square Error value [5].M. FerniUkrit et al. proposed compression method combines Super-Spatial Structure Prediction with inter-frame coding to accomplish higher compression ratio. Primarily; the super spatial structure prediction algorithm is applied with the fast block-matching process which includes diamond search scheme. Inadditionally increasing the compression ratio propose a new scheme Head Code Compression. Experimental results of our proposed Composite algorithm for medical image sequences achieve 25\% more reduction than the prior arts [6].Duc Minh Pham et al. they paper presented an inventive architecture and protocol for energy proficientprocessing and communication of images over wireless sensor networks. The proposed hardware design is optimized to accomplish high speed image compression with minimum hardware constraint and low power consumption. An efficient and trustworthy transmission protocol for compressed image is also presented. It integrates an effective image packet queue control strategy to reduce packet error rate and increase image transmission throughput. An experimental results show the effectiveness of these new approaches to make image compression and communication over wireless sensor networks feasible, reliable and efficient [7].KhaledSahnoun et al. has presented a new codingmethod for satellite images. In first the image will bedownloaded followed by a fast Fourier transform FFT. Theresult gained after FFT processing suffers a scalarquantization (SQ). The results obtained after the quantizationphase are encoded using entropy encoding. This method hasbeen tested on satellite image and Lena image. Subsequently todecompress theimages were reconstructed authentically andmemory space required for storage has been reduced by morethan $80 \%$ [8].JiantaoZhou et al. Designed a highly efficient image encryption-thencompression (ETC) system, where both lossless and lossy compressions are measured. The proposed image encryption method operated in the forecast error domain is shown to be able to supply a reasonably high level of security. This also demonstrates that an arithmetic coding-based approach can be exploited to efficiently compress the encrypted images. Most notably; the proposed compression method applied to encrypted images is only slightly inferior in terms of compression efficiency than the state-of-the-art lossless/lossy image coders which take new unencrypted images as inputs. In contrast most of the existing ETC solutions induce significant penalty on the compression efficiency [9].Hong-jun $\mathbf{L i}$ et al. proposed an image compression algorithm based on grey relational theory in wavelet domain. Theyuse the character of wavelet coefficients and pertains the grey relational theory in coefficients relational description and then suggest an image compression method via grey relational theory. They classified the coefficients according to their characters in different domains and create the sparse representation method under different types of coefficients. The algorithm reduces the computational complexity and improves the ability of image sparse representation. It accomplishes an efficient way of image compression. Itshows that the proposed compression algorithm based on grey relational theory is superior to the other algorithms both in the visual quality and PSNR [10].Kai-jen Cheng et al.This paper proposes a lossless to lossy compressionscheme for hyper-spectral images based on dual-tree BinaryEmbedded Zerotree Wavelet (BEZW) algorithm. The algorithmadapts Karhunen-Loève Transform and Discrete Wavelet Transformto achieve 3-D integer reversible hybrid transform andde-correlate spectral and spatial data. Since statistics of the hyper-spectralimage are not symmetrical, the asymmetrical dual-treestructure is introduced. The 3-D BEZW algorithm compresses hyper-spectralimages by implementing progressive bitplane coding.The lossless and lossy compression performance is compared withother state-ofthe-art predictive coding and transform-based codingalgorithms on Airborne Visible/Infrared Imaging Spectrometerimages. Results show that the 3-D-BEZW lossless compressionperformance is comparable with the best predictive algorithms, while its computational cost is comparable with those of transform basedalgorithms [11].

\subsection{D DT-CWT}

The 2D DT-CWT also more selectively discriminates features of various orientations. Whereas the critically decimated 2D DWT outputs three orientation selective sub-bands per level conveying image features oriented at the angles of $90^{\circ}, \pm 45^{\circ}$, and $0^{\circ}$, the 2D DT-CWT produces six directional sub bands per level to reveal the details of an image in $\pm 15^{\circ}, \pm 45^{\circ}$ and $\pm 75^{\circ}$ directions with 4:1 redundancy [19].

\section{IMAGE COMPRESSION TECHNIQUES}

Image compression techniques are mostly classified into two categories depending whether or not an exact replica of the original image could be reconstructed using the compressed image.

\section{Lossless technique}

2. Lossy technique

\subsection{Lossless Compression Techniques}

In lossless compression techniques the original image can be absolutely recovered from the compressed (encoded) image. These are also known as noiseless because they do not add noise to the signal (image).It is also called entropy coding 
because it uses decomposition techniques to eliminate/minimize redundancy. The lossless compression is used simply for a few applications with strict requirements such as medical imaging. Subsequent techniques are incorporated in lossless compression:

1. Run length encoding

2. Huffman encoding

3. LZW coding

4. Area coding

\section{Run Length Encoding}

This method is a simple lossless compression method. It is mainly used for sequential data. This is most useful on data that contains repetitive information. This method will replace matching symbols. These identical symbols are known as runs. They are substituted by shorter symbols. This method is supported by most bitmap file formatslikewaise TIFF, BMP, and PCX.

RLE $[12,13]$ is an appropriate for compressing any type of data despite of its information content but the content of the data will affect the compression ratio achieved by RLE. Even thoughthe most RLE algorithms cannot accomplish the high compression ratios of the more advanced compression methods, RLE is both uncomplicated to implement and rapid to implement making it a good alternative to either using a complex compression algorithm or leaving your image data uncompressed. There are a variety of variants of run-length encoding. Image data is usually run-length encoded in sequential processes that behaves the image data as a 1-D stream rather than as a 2-D map of data. In sequential processing, a bitmap is encoded starting at the upper left corner and proceeding from left to right across each scan line (the $\mathrm{X}$ axis) to the bottom right corner of the bitmap But alternative RLE schemes can also be written to encode data down the length of a bitmap (the $\mathrm{Y}$ axis) along the columns to encode a bitmap into 2-D tiles or even to encode pixels on a diagonal in a zigzag fashion Odd RLE variants such as this last one might be used in extremely specialized applications but are usually quite rare.

\section{Huffman encoding}

Huffman coding can generate a code that is as close as possible to the minimum bound, the entropy. This method results in variable length coding. For complex images, Huffman code alone will reduce the file size by 10 to $50 \%$. By removing irrelevant information first, file size reduction is possible [14].

\section{LZW coding}

LZW (Lempel- Ziv - Welch) coding can be static or dynamic which is a dictionary based coding. In static dictionary coding dictionary is fixed during the encoding and decoding method. On the other hand in dynamic dictionary coding the dictionary is updated on fly. The computer industry is extensively using LZW [14]. It is also implemented as compress command on UNIX.

\section{Area coding}

Area coding is an improved form of run length coding which reflects the two dimensional character of images. It is a vital advancement over the other lossless methods. It does not make much of a meaning to infer the coding of an image as a chronological stream as it is in actuality an array of sequences building up a two dimensional object. The idea behind this is to find the rectangular regions with the same features. These rectangular regions are coded in aexpressive form as an constituent with two points and a certain structure. Area coding $[14]$ is highly effective and it can give high compression ratio but the limitation being non-linear in nature, which prevents the implementation in hardware.

\subsection{Lossy Compression Techniques}

Lossy compression technique provides higher compression ratio than lossless compression. In this method, the compression ratio is high; the decompressed image is not exactly identical to the original image, but close to it. Different types of lossy compression techniques are extensively used characterized by the eminence of the reconstructed images and its sufficiency for applications. The quantization process applied in lossy compression technique results in loss of information. After quantization, entropy coding is done like lossless compression. The decoding is a reverse process. The entropy decoding is applied to compressed data to get the quantized data. Dequantization is applied to it andlastly the inverse transformation is performed to obtain the reconstructed image. The methods that fall under lossy compression method are listed in figure 2:

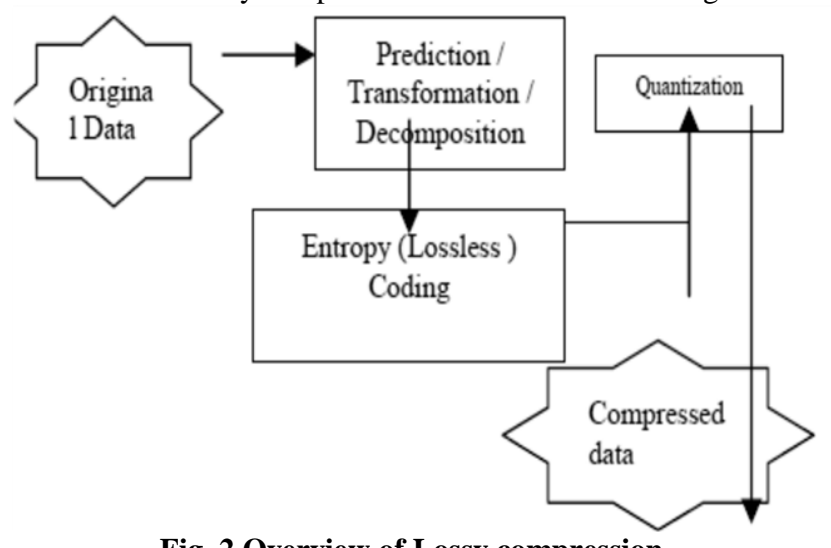

Fig. 2 Overview of Lossy compression

Lossy compression techniques comprises following schemes:

1. Transformation coding

2. Vector quantization

3. Fractal coding

4. Block Truncation Coding

5. Sub-band coding

\section{Transform coding}

It is a category of compression for natural data like photographic images. It will result a low quality output of original image. It is a core method recommended by jpeg. Transform coding is used to change spatial image pixel values to convert coefficient values. Becauseof this is a linear process and no information is lost the number of coefficients produced is equal to the number of pixels transformed. Various types of transforms have been tried for picture codingtogether with example Fourier Karhonen-Loeve, Walsh-Hadamard, lapped orthogonal, discrete cosine (DCT), and freshly wavelets.

\section{Vector Quantization}

One of the most common methods to compress images is to code them with the vector quantization (VQ) techniques. The theory of the VQ techniques is simple. At first, the image is splitted into square blocks of $n \times n$ pixels, for example $4 \times 4$ or $8 \times$ 8 ; each block is considered as a vector in a 16- or 64 dimensional space, respectively. Second, a limited number ( 1) of vectors (code words) in this space is selected in order to approximate as much as possible the distribution of the initial vectors extracted from the image; in other words, more code words will be placed in the region of the space where there are more points in the initial distribution (image), and vice versa. 
Third, each vector from the original image is replaced by its nearest codeword (usually according to a second-order distance measure). Finally, in a transmission scheme, the index of the codeword is transmitted instead of the codeword itself; the compression is achieved if the number of bits used to transmit this index $(\log 21)$ is less than the number of initial bits of the block ( $n \times n \times m$ if $m$ is the resolution of each pixel) [15].

\section{Fractal coding}

The basic idea behind fractal compression is to represent an image as a fixed point of IFSs. An input image could be represented by a series of IFS codes rather than pixels.

In this way, an amazing compression ratio could be achieved. Therefore the main aim of fractal algorithm is to find fractals, which could best approximate the original image and then represent it as a set of affine transformations. Its excellent compression property and the advantage of very fast decompression have created a great interest in its field. A twodimensional image is represented mathematically as $\mathrm{z}=\mathrm{f}(\mathrm{x}, \mathrm{y})$, where $\mathrm{f}(\mathrm{x}, \mathrm{y})$ represents the grey level with 0 being black and 1 being white at the point $(\mathrm{x}, \mathrm{y})$ in an image. I denotes the close interval $\left[\begin{array}{ll}0 & 1\end{array}\right]$. On applying affine transformation ' $\mathrm{W}$ ' on to image ' $\mathrm{f}$ ', we get a transformed image W( $\mathrm{f}$ ). W constantly moves points closer together as it is contractive. Affine transformations are blends of scaling, rotations and transformation of the coordinate axes in n-dimensional space, which always map squares to parallelograms. The general form of affine transformation is given by:

$$
W\left[\begin{array}{l}
x \\
y
\end{array}\right]=\left[\begin{array}{ll}
a & b \\
c & d
\end{array}\right]\left[\begin{array}{l}
x \\
y
\end{array}\right]+\left[\begin{array}{l}
e \\
r
\end{array}\right]=\left[\begin{array}{l}
a x+b y+e \\
c x+d y+f
\end{array}\right]
$$

If the translations, scaling and rotations are known inadvance, then the coefficients may be calculated by: $a=\operatorname{ros} \theta ; b=-\sin j$; $\mathrm{c}=\mathrm{r} \sin \theta ; \mathrm{d}=\mathrm{s} \cos \mathrm{j}$ where the scaling factors are $\mathrm{r}$ and $\mathrm{s}$, the angle of rotations are $\theta$ and $j$ and the translations are e and $f$. The transformation is found suitable for encoding grey-scale images as a three-dimensional image, where coordinates such as $\mathrm{x}$ and $\mathrm{y}$ and intensity such as $\mathrm{z}$ is given in (2), where si controls the contrast and oi controls the brightness of transformation.

$$
w_{i}\left[\begin{array}{l}
x \\
y \\
z
\end{array}\right]=\left[\begin{array}{ccc}
a i & b i & 0 \\
c i & d i & 0 \\
0 & 0 & s i
\end{array}\right]\left[\begin{array}{c}
x \\
y \\
z
\end{array}\right]+\left[\begin{array}{c}
e i \\
f i \\
o i
\end{array}\right]
$$

According to the contractive mapping fixed theorem, if the transformation is contractive then applying transformation repeatedly starting with any initial point would lead to convergence to a unique fixed point. If $\mathrm{X}$ is a complex metric space and $\mathrm{W}: \mathrm{X}->\mathrm{X}$ is contractive then $\mathrm{W}$ has a unique fixed point. Precisely, the collection of transformation defines an image.

\section{Block truncation coding}

The principle applied here is that the image is divided into non overlapping blocks of pixels. The mean of the pixel values in the block (threshold) and reconstruction values are determined for each block. Then a bitmap of the block is created by replacing all pixels whose values are greater than or equal (less than) to the threshold by zero or one. Then for each segment (group of $1 \mathrm{~s}$ and $0 \mathrm{~s}$ ) in the bitmap the renovation value is strong-minded. This is the average of the values of the corresponding pixels in the original block.

\section{Sub Band Coding}

In the sub band coding the image is analyzed and find the components containing frequencies in different bands, the sub bands. Then the quantization and coding are performed for each sub-band. The main advantage of this coding is that quantization and coding for each sub-band can be designed separately.

\subsection{Performance Metrics}

There are two performance parameters [18] are used to measure the performance of the image compression algorithms. One is PSNR (peak signal to noise ratio) and second is Mean square error (MSE). PSNR is the measurement of the peak error between the compressed image and original image. The higher the PSNR contains enhanced quality of image. To calculate the PSNR first of all MSE (mean square error) is computed. Mean Square Error (MSE) is the cumulative difference between the compressed image and original image. Small amount of MSE reduce the error and improves image quality.

MSE $=\frac{1}{M \amalg N} \sum_{y=1}^{M} \sum_{x=1}^{N}\left[I(x, y)-I^{\prime}(x, y)\right]^{2}$

In the previous equation, $\mathrm{M}$ and $\mathrm{N}$ are the number of rows and columns in the input images. The PSNR is computed from following equation:

$\mathrm{PSNR}=20 * \log 10(255 / \operatorname{sqrt}(\mathrm{MSE}))$ eq(4)

Table 1: Advantages and Disadvantages of Compression technique

\begin{tabular}{|l|l|l|}
\hline \multicolumn{1}{|c|}{ Method } & \multicolumn{1}{|c|}{ Advantages } & \multicolumn{1}{c|}{ Disadvantages } \\
\hline SIFT & $\begin{array}{l}\text { Stability, Accuracy, } \\
\text { Reliability }\end{array}$ & Key Localization \\
\hline PCA-DCT & $\begin{array}{l}\text { Very efficient and Fast } \\
\text { to compute }\end{array}$ & Noise Increases \\
\hline RBF & $\begin{array}{l}\text { Free from Local } \\
\text { Minima }\end{array}$ & Very Slow \\
\hline FFT & $\begin{array}{l}\text { Less } \\
\text { encoding/decoding } \\
\text { time }\end{array}$ & Sever Block effect \\
\hline ETC & $\begin{array}{l}\text { High level Security } \\
\text { BEZW }\end{array}$ & $\begin{array}{l}\text { Less efficient in } \\
\text { compression }\end{array}$ \\
\hline
\end{tabular}

\section{CONCLUSION}

In the field of multimedia image compressionis one of the tasks and for reducing or compressing it there has been developed various techniques. In this paper literature of some of the techniques is describedbut someof them are not good for compressing the size of image data while some has but by that blur image if formed so in future determine such method which compresses accordingly and image also must be clear.

\section{REFERENCES}

[1] S. Narasimhulu1, Dr. T. Ramashri 2012, "Gray-Scale Image Compression Using DWT-SPIHT Algorithm", 
International Journal of Engineering Research and Applications (IJERA) ISSN: 2248-9622 www.ijera.com Vol. 2, Issue 4, pp.902-905.

[2] Liu,C.P.; Poularikas,A.D.” 1996, A New Subband Coding Technique Using (JPEG) DCT for Image Compression," IEEE Trans. on Image processing, pp.317-321.

[3] HuanjingYue, Xiaoyan Sun, Feng Wu, Jingyu Yang, "SIFT-BASED IMAGE COMPRESSION", IEEE International Conference on Multimedia and Expo.

[4] Merav Huber-Lerner, OferHadar, Stanley R. Rotman, Revital Huber-Shalem 2012, "Compression of Hyperspectral Images Containing a Sub-Pixel Target", IEEE 27th Convention of Electrical and Electronics Engineers in Israel.

[5] G BoopathiDr.S.Arockiasamy 2012, "Image Compression: Wavelet Transform using Radial Basis Function (RBF) Neural Network", India Conference (INDICON), Annual IEEE, Page(s): 340 - 344 Print ISBN: 978-1-4673-2270-6.

[6] MFerniUkrit, G.R.Suresh 2013, "Effective Lossless Compression for Medical Image Sequences Using Composite Algorithm", International Conferenceon Circuits, Power and Computing Technologies In proceeding IEEExplore, 978-1-4673-4922-2.

[7] Duc Minh Pham, Syed Mahfuzul Aziz 2013, “ An energy efficient image compression scheme for wireless sensor networks", Intelligent Sensors, Sensor Networks and Information Processing,IEEE Eighth International Conference on Melbourne, VIC, Page(s): 260 - 264 Print ISBN: 978-1-4673-5499-8

[8] KhaledSahnoun, NoureddineBenabadji 2013, “On-Board Satellite Image Compression Using The Fourier Transform And Huffman Coding", Computer and Information Technology (WCCIT),World Congress on Sousse, Page(s): 1 - 5 Print ISBN: 978-1-4799-0460-0.

[9] Jiantao Zhou, Xianming Liu, Oscar C. Au, Yuan Yan Tang 2014, "Designing an Efficient Image Encryption-ThenCompression System via Prediction Error Clustering and
Random Permutation", IEEE Transactions On Information Forensics And Security, Vol. 9, No. 1.

[10] Hong-jun Li, Zheng-guangXie, Wei Hu 2013, “An Image Compression Method using Sparse Representation and Grey Relation", Grey Systems and Intelligent Services, IEEE International Conference, Macao, Page(s): 53 - 56, ISSN:2166-9430 in proceeding of IEEE xplore.

[11] Kai-jen Cheng, Jeffrey Dill 2014, "Lossless to Lossy DualTree BEZW Compression for Hyper-spectral Images", IEEE Transactions on Geosciences and Remote Sensing, IEEE.

[12] S.R. Kodituwakku,U. S. Amarasinghe 2004,” Comparison of Lossless Data Compression Algorithms for Text Data",Indian Journal of Computer Science and Engineering Vol 1 No 4 416-425,IJCSE.

[13] [13] Blelloch, E. 2002, "Introduction to Data Compression", Computer Science Department, Carnegie Mellon University.

[14] Woods, R. C, "Digital Image processing". New Delhi: Pearson Prentice Hall, Third Edition, Low price edition, Pages 1-904.

[15] G. M. Padmaja, P. Nirupama 2012, "Analysis of Various Image Compression Techniques", ARPN Journal of Science and Technology, VOL. 2, NO. 4, ISSN 2225-7217.

[16] SridharanBhavani, KepannaGowderThanushkodi 2013, "Comparison of fractal coding methods for medical image compression", IET Image Process., Vol. 7, Iss. 7, pp. 686693.

[17] Mr.Chandresh K Parmar, 2prof.Kruti Pancholi "A Review on Image Compression techniques" Journal of Information, Knowledge and Research in Electrical Engineering.

[18] Vijayshri, C. and S. Ajay 2010, Review of a novel technique: Fractal image compression. Int. J. Eng. Techn., 1(1): $53-56$

[19] E. Hostalkova, A. Prochazka, "Hilbert Transform Pairs of Wavelet Bases", internet link. 